\title{
A PRESSÃO ARTERIAL ESTÁ SENDO MEDIDA?
}

\section{IS THE BLOOD PRESSURE BEING MEASURED?}

\author{
Débora C. Alavarce* \\ Angela Maria Geraldo Pierin** \\ Décio Mion $J r^{* * *}$
}

ALAVARCE, D. C.et al. A pressão arterial está sendo medida? Rev.Esc.Enf.USP, v. 34, n. 1, p. 84-90, mar. 2000.

\section{RESUMO}

\begin{abstract}
A medida da pressão arterial faz parte do exame físico e deve ser realizada em toda consulta médica. Para avaliar se a pressão arterial é medida nas consultas médicas, foram consultados 500 prontuários de pacientes de primeira consulta ambulatorial, sendo 335 (67\%) provenientes de clínicas médicas e cirúrgicas e 165 (33\%) das clínicas ginecológica e obstétrica. A pressão arterial foi anotada em $39 \%$ das consultas realizadas $(135 \pm 32 / 85 \pm 19 \mathrm{~mm} \mathrm{Hg})$, sendo que em $11 \%$ destas anotações a pressão diastólica estava acima de $90 \mathrm{~mm} \mathrm{Hg}$. Em relação ao diagnóstico prévio de hipertensão arterial verificou-se que: a) em $62 \%$ dos prontuários não havia esta informação; b) $20 \%$ referiram ter hipertensão, e em $79 \%$ destes a pressão arterial foi anotada; e c) 18\% referiram não ter hipertensão e $46 \%$ destes tiveram registro da pressão arterial. Portanto, a maior parte dos pacientes não teve sua pressão arterial anotada.
\end{abstract}

PALAVRAS-CHAVES: Hipertensão. Pressão arterial.

\section{ASTRACT}

The blood pressure measure is part of the physical exam and it should be accomplished in every medical consultation. To verify the blood pressure measured in the accomplished medical consultations record of first they were consulted it consults 500 patient's ambulatorial being 335 (67\%) coming of medical and surgical clinics and 165 (33\%) of the gynecological and obstetric clinics. The blood pressure was written down in 39\% of the accomplished consultations $(135+32 \mathrm{~mm} \mathrm{Hg} 85+19 \mathrm{~mm}$ $\mathrm{Hg}$ ), and $11 \%$ of this annotations the pressure diastólica was above $90 \mathrm{~mm} \mathrm{Hg}$. In relation to the previous diagnosis of hypertension it was verified that: a) in $62 \%$ of the records there was not this information; b) $20 \%$ referred to have hypertension, and in $79 \%$ of these the arterial pressure was scored; and c) $18 \%$ referred not to have hypertension and $46 \%$ of these had registration of the arterial pressure. Therefore, most of the patients didn't have its logged arterial pressure.

KEYWORDS: Hypertension. Blood pressure.

\section{INTRODUÇÃO}

A medida da pressão arterial é um procedimento simples, fácil de ser executado e deve ser realizada em todas as avaliações de saúde independente da especialidade do atendimento".

O enfermeiro desempenha papel importante na medida da pressão arterial, seja na detecção precoce da doença - hipertensão arterial ou no controle e acompanhamento dos hipertensos, bem como na orientação destas pessoas.

Os valores obtidos pela medida da pressão arterial apresentam correlação direta com a morbidade e mortalidade cardiovascular. O método indireto com técnica auscultatória é o meio mais utilizado para avaliação da medida da pressão arterial. Portanto, decisões relativas ao diagnóstico, prognóstico e tratamento da hipertensão arterial dependem desta avaliação3.

A preocupação em medir a pressão arterial remonta a séculos atrás. Esta busca aponta referências na literatura de 1733 na Inglaterra, quando o reverendo

\footnotetext{
* Aluna de graduação da Escola de Enfermagem da USP. E-mail: dalavarce@zipmail.com.br

** Profa. Livre Docente. Escola de Enfermagem da USP - Dep. Enfermagem Médico Cirúrgica. E-mail: angela_pierin@hotmail.com

*** Médico Chefe da Liga de Hipertensão do Hospital das Clinicas da Faculdade Medicina da USP -
} 
Stephen Hales introduziu um tubo na carótida de uma égua, na tentativa de visualizar as oscilações do pulso sangüíneo e avaliar os sincronismo dos batimentos cardíacos, causando surpresa ao pesquisador a altura atingida pela coluna líquida de $290 \mathrm{~cm}$. Tal experimento caracterizou o primeiro registro direto da pressão arterial que se tem conhecimento. Mas foi no final do século seguinte, em 1896, que Scipione Riva Rocci, em Torino, Itália, passou da fase puramente experimental para aplicação clínica da medida da pressão arterial, idealizando o primeiro esfigmomanômetro de coluna de mercúrio com um manguito de 4,5 $\mathrm{m}$ de largura 4,5

Em 1904 o russo Nicolas Sergievic Korotkoff, ao verificar as alterações causadas pela turbulência do fluxo sangüineo durante a inflação e a deflação da bolsa de borracha, em decorrência das diferentes pressões provocadas no interior do vaso, sugeriu a possibilidade de auscultar o pulso durante a compressão da artéria. Outra contribuição de Korotkoff foi a determinação da pressão diastólica ${ }^{4,5}$.

Decorridos cerca de um século, o método indireto com técnica auscultatória é o recurso mais utilizado para a medida da pressão arterial. E um procedimento simples, fácil de ser realizado e quando executado de maneira correta provê valores realmente fidedignos de avaliação dos níveis tensionais para o diagnóstico e tratamento da hipertensão arterial.

A hipertensão arterial é uma doença altamente prevalente em nosso meio, acomete cerca de 15 a $20 \%$ as pessoas com idade acima de 18 anos, podendo atingir índices de $50 \%$ nos idosos ${ }^{1,2}$

A disponibilidade de informações sobre a prevalência da hipertensão é geralmente dificultada, pois nas relações de causa mortis ela raramente aparece como causa básica, apesar de se relacionar com $80 \%$ dos casos de doenças cérebro vascular, quase $50 \%$ dos casos de cardiopatia isquêmica e uma boa proporção de doenças do coração e do sistema circulatórios.

Salienta-se ainda, a importância da medida da pressão arterial na detecção precoce da doença. A hipertensão arterial quando oportuna e adequadamente tratada reduz a morbidade e mortalidade cardiovascular 7,8. Dados de 1988 do Ministério da Saúde indicam que a hipertensão arterial foi a segunda maior causa de internação $(21,4 \%)$ e como causa isolada foi o primeiro motivo de aposentadoria no país, no período de 1980/1986 9,10.

Do ponto de vista sócio econômico, os gastos com hipertensão merecem destaque, pois estima-se que as aposentadorias por hipertensão ocorrem, em média, 12 anos antes do tempo previsto".

Apesar da medida da pressão arterial ser um procedimento relativamente simples e um parâmetro imprescindivel para avaliação cardiocirculatória, não se garante que a pressão arterial seja medida rotineiramente.
Considerando, então, a magnitude da hipertensão arterial e a importância da detecção precoce da doença, o presente estudo teve por finalidade verificar se a pressão arterial está sendo medida nos atendimentos de saúde em diferentes especialidades num hospital escola do município de São Paulo e se operacionaliza nos seguintes objetivos:

1. Identificar a realização da medida da pressão arterial, avaliando-se a anotação em prontuários de pacientes atendidos em consultas de diferentes especialidades.

2. Relacionar a realização ou não da medida da pressão arterial com história anterior de hipertensão arterial.

3. Verificar a influência das variáveis sexo e idade com a realização da medida da pressão arterial.

\section{MATERIAL E MÉTODO}

\section{Local de realização do estudo}

O estudo foi realizado no ambulatório de um hospital geral e de ensino da cidade de São Paulo.

Foi solicitada à instituição autorização para realização do estudo e após a anuência é que se deu a coleta de dados.

\section{Critérios de seleção}

Para realização do estudo, foram analisados todos prontuários de pacientes atendidos em primeira consulta de diferentes especialidades médicas, nos dias de coleta de dados, até que se totalizasse 500 prontuários abarcando todas as especialidades de atendimento no ambulatório. Os prontuários analisados se distribuíram da seguinte forma: 335 (67\%) pertenciam a 24 clínicas médicas e cirúrgicas, dos quais 8,5\% pertenciam à Liga de Hipertensão, os 165 $(33 \%)$ restantes referiam-se às clínicas de ginecologia e obstetrícia.

\section{Coleta de dados}

Os dados foram coletados utilizando-se instrumento específico (anexo) composto das partes relacionadas a seguir.

Parte 1: Identificação caracterização da clínica, nome do paciente, número de registro, sexo, idade, cor e procedência.

Parte 2: Medida da pressão arterial - teve por finalidade avaliar a realização ou não da medida da pressão arterial por meio das anotações existentes, foram anotados todos os valores constantes na primeira consulta. 
Parte 3: Histórico de hipertensão arterial - o objetivo foi identificar se foram pesquisados antecedentes familiares de hipertensão arterial e em caso afirmativo qual o grau de parentesco.

\section{Tratamento dos dados}

Os dados foram computados em programa específico, D'BASE III e EPI-INFO.

Por se tratar de um estudo descritivo exploratório, os dados foram avaliados considerando-se os indices absolutos e percentuais, para atender os objetivos da pesquisa e apresentados na forma de figuras.

\section{RESULTADOS}

\section{A medida da pressão arterial}

Os dados do presente estudo indicam que 61\% (305) das consultas não tiveram a pressão arterial anotada, portanto, em apenas 39\% havia anotação de valores da pressão arterial. Os valores encontrados para as pressões sistólica e diastólica (média \pm desvio padrão) foram de $135 \pm 32$ e $85 \pm 19 \mathrm{~mm} \mathrm{Hg}$, respectivamente. As consultas (39\%) que continham anotações da pressão arterial, em $21 \%$ das anotações, os niveis de pressão diastólica foram acima de $90 \mathrm{~mm} \mathrm{Hg}$. Verificou-se ainda, que 8,5\% das pressões arteriais anotadas referem-se à Liga de Hipertensão do hospital onde o estudo foi realizado. (Figura 1)

Figura 1 - Anotação da medida da pressão arterial nas consultas analisadas. São Paulo, 1997.

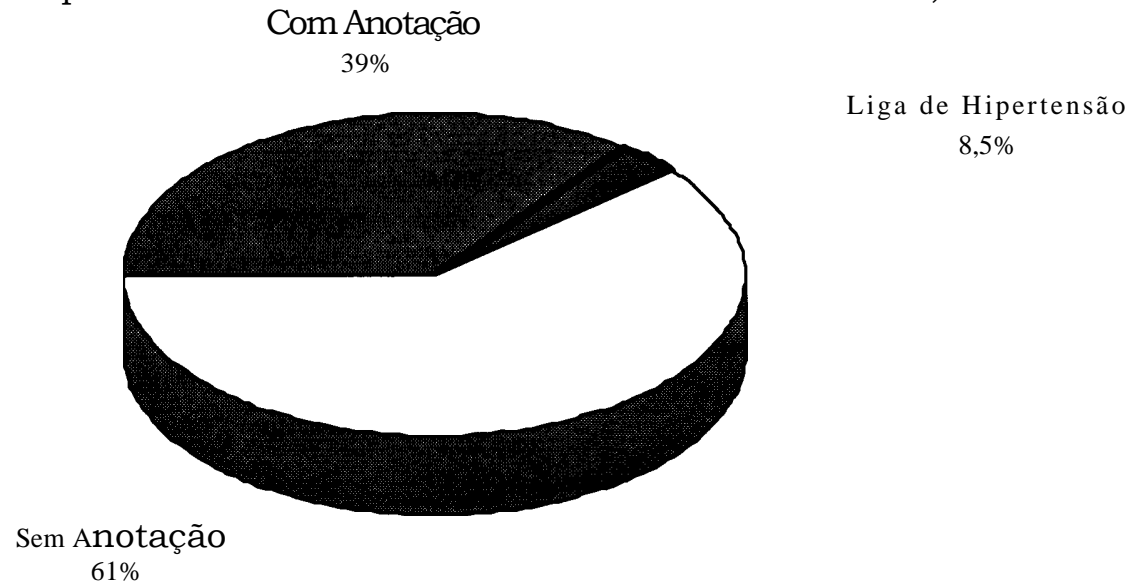

Caracterização das variáveis estruturais dos pacientes cujos prontuários foram avaliados.

Os pacientes das consultas, em que se verificou a existência ou não de anotação da medida da pressão arterial, possuíam idade de $38 \pm 18$ anos (média \pm desvio padrão).

Observou-se as seguintes freqüências de anotação da medida da pressão arterial de Acordo com as faixas etárias: $21 \%$ nos pacientes com menos de 20 anos, 39\% naqueles com idade entre 21 e 40 anos, $46 \%$ entre 41 e 60 anos e $33 \%$ nos pacientes com mais de 60 ANOS.(FIGURA 2)

Figura 2 - Distribuição dos pacientes de acordo com a idade e anotação de medida da pressão arterial. São Paulo, 1997.

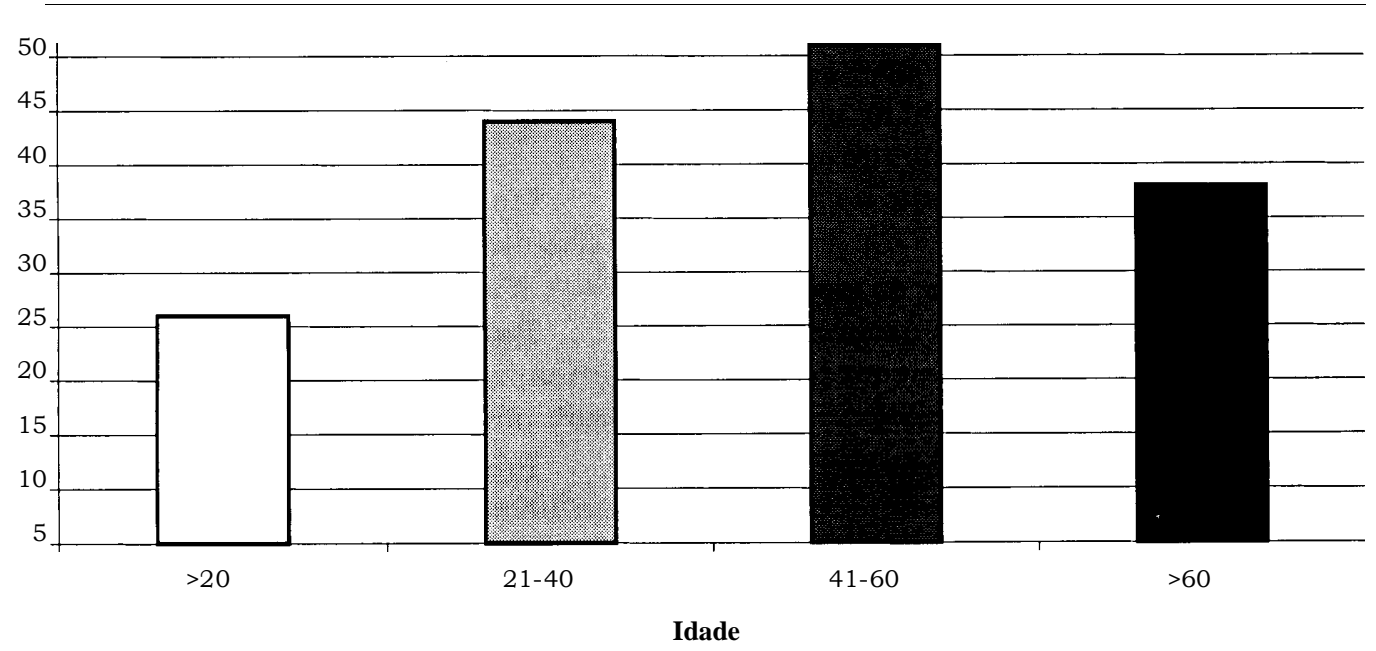


Quanto ao sexo, $75 \%$ das consultas analisadas eram de mulheres e destas $45 \%$ tiveram sua pressãoarterial anotada. Dos pacientes do sexo masculino $25 \%$ tiveram seus valores de pressão arterial anotados.

Figura 3 - Distribuição dos pacientes segundo sexo e a anotação da medida da pressão arterial. São Paulo, 1997.

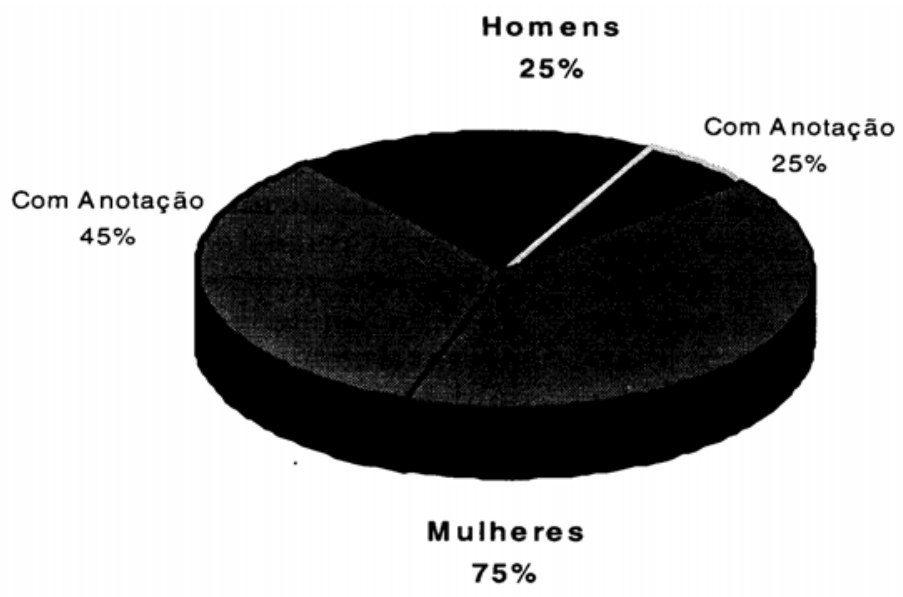

\section{História de hipertensão}

Das 500 primeiras consultas avaliadas, $62 \%$ não continham dados sobre antecedente familiar para hipertensão, $20 \%$ referiram antecedentes e $18 \%$ apontaram ausência de antecedentes para hipertensão. Nos pacientes com história de hipertensão, houve anotação da medida da pressão arterial em $79 \%$. No grupo de pacientes que negou história de hipertensão familiar, $46 \%$ tiveram pressão arterial anotada. (Figura 4) Figura 4 - Distribuição dos pacientes de acordo com a história de hipertensão e a anotação da medida da pressão $\operatorname{ar}^{1-\cdots-1} \sim \sim-n+1-1 n n \rightarrow$

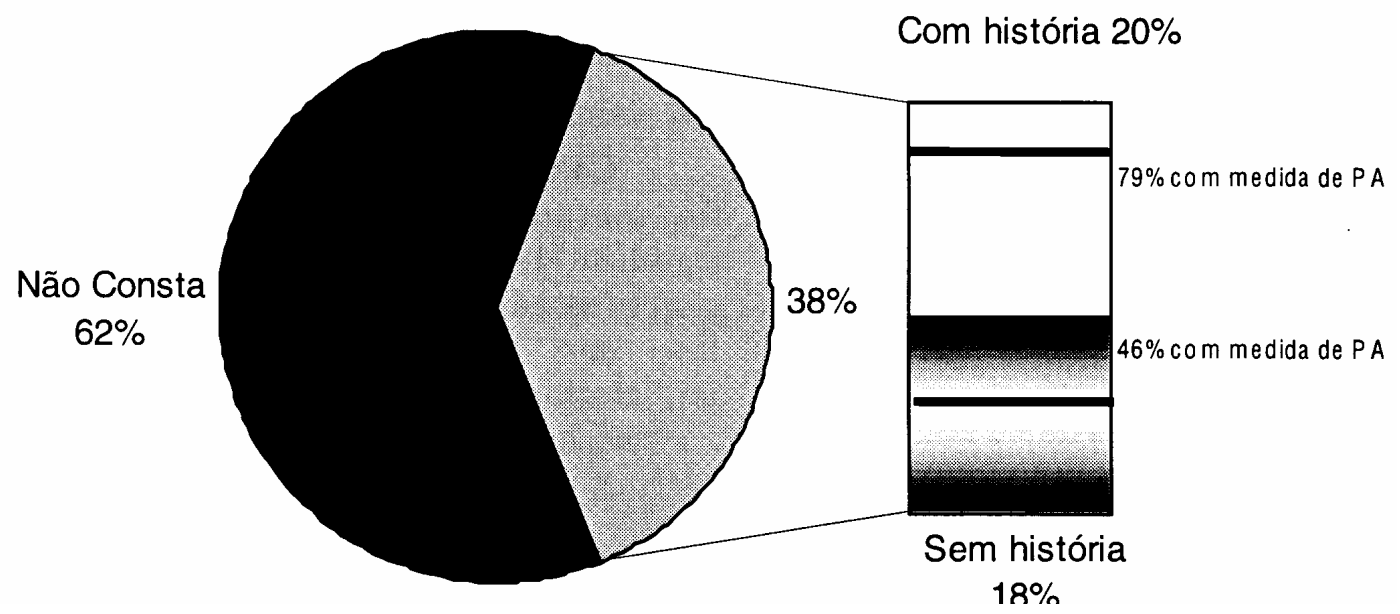

O instrumento de coletas de dados previa identificação do paciente quanto à etnia e procedência e em relação à medida da pressão arterial posição do paciente e tipo de aparelho. Porém, estes itens não foram analisadas pela inexistência no prontuário de dados a respeito.

\section{DISCUSSÃO}

O principal achado do presente estudo foi que apenas 39\% das consultas analisadas continham anotação da medida da pressão arterial. Considerando que o registro nó prontuário do paciente reflete as ações da equipe de saúde, poderia se supor que a efetiva realização da avaliação dos níveis tensionais guarda relação direta com a existência de anotação.

Portanto, número significativo de pessoas poderia não ter dentro da avaliação cardiocirculatória o parâmetro vital, pressão arterial, mensurado.

A medida da pressão arterial é um procedimento fácil, simples de ser realizado e é o recurso utilizado para o diagnóstico da hipertensão arterial. A hipertensão arterial é uma doença altamente prevalente em nosso meio, atinge cerca de 15 a 20\% as pessoas adultas, sendo que nos idosos estes índices chegam a 50\% ${ }^{1,2}$. É uma doença que apresenta correlação direta com morbi e mortalidade por doenças cardiovasculares, que por sua vez ocupa o primeiro lugar no perfil epidemiológico 
em nosso meio. No presente estudo, cerca de 60 pessoas poderiam não ter sido diagnosticadas pela não realização da medida da pressão arterial.

Estudo patrocinado pelo Banco Mundial e pela Organização Mundial de Saúde, que avaliou "anos ajustados de incapacidade e de vida" para identificar o impacto das doenças e das intervenções sobre estado de saúde das populações, expôs que a carga global representada pela hipertensão arterial de um total de mais de 20 milhões de mortes mundiais por todas as causas, é de aproximadamente 3 milhões ${ }^{6}$. Segundo dados do Ministério da Saúde sobre as doenças do aparelho circulatório registrados em 1993 no Brasil, a doença hipertensão arterial ficou em $4^{\circ}$ lugar com $7 \%$ do total 10.

Estudo realizado por LESSA et al. 10 no ano de 1982 na cidade de Salvador identificou que em apenas $18,7 \%$ dos adultos atendidos em primeira consulta nos serviços médicos de diferentes modalidades assistenciais, tiveram sua pressão arterial medida e naqueles em que a pressão arterial foi avaliada, 22\% apresentaram níveis acima dos valores de normalidade; concluindo que dos $82 \%$ restantes dada a prevalência da hipertensão arterial, pelo menos $10 \%$ seriam hipertensos e não foram identificados pela não avaliação da pressão arterial $^{\circ}$. Dando seguimento ao estudo, Lessa em 1991 avaliou os prontuários de primeira consulta dos mesmo serviços analisados em 1982 verificando uma freqüência de anotações de $35,9 \%$ demonstrando um aumento de $92 \%$ na freqüência quando comparada aos $18,7 \%$ observados em $1982^{10}$. Embora este incremento pareça satisfatório, ainda se evidencia a baixa freqüência de realização das medidas de pressão arterial.

Considerando os resultados da presente investigação reforça-se o fato de que a pressão arterial está sendo medida numa parcela muito pequena da população. Apesar da amostra incluir prontuários da Liga de Hipertensão, nos quais necessariamente devem ser incluídos dados da medida da pressão arterial. Este fato é preocupante, dada a relevância que acompanha o tema, o alto índice de morbi-mortalidade por doenças cardiocirculatórias e correlação direta com hipertensão arterial e conhecendo-se os prejuízos que esta doença traz, tanto em qualidade de vida quanto em custo sócioeconômico para o país e para a população. Conhecendose hoje os métodos, farmacológico e não farmacológico, para o tratamento da hipertensão; a importância e os beneficios do diagnóstico precoce; e a relativa facilidade em executar a medida da pressão arterial, os dados aqui apresentados merecem atenção de todos os profissionais da área da saúde.

Outro ponto que merece destaque foi que em relação à idade a menor freqüência $(21 \%)$ de anotação da medida da pressão arterial ocorreu naqueles com menos de 20 anos. A não realização da medida da pressão arterial em pessoas jovens compromete o diagnóstico precoce da doença com maior tempo de exposição aos efeitos deletéricos da hipertensão arterial. Nas pessoas com mais de 60 anos, a medida da pressão arterial foi anotada em 33\%, o que também merece atenção, tendo em vista o aumento da prevalência da hipertensão arterial nos idosos.

Sabe-se hoje, que a pressão arterial eleva-se com a idade, embora varie fisiologicamente de um indivíduo para outro de momento a momento. As variações das pressões sistólica e diastólica não correm de forma paralela, pois a pressão sistólica aumenta consistentemente com a idade e a pressão diastólica mostra um aumento até a meia-idade, quando tende a se nivelar, para baixar no final, provavelmente por um fenômeno de seleção, onde os indivíduos com pressão diastólica mais elevada vão sendo excluídos por morte mais precoces. Avaliandose o número baixo de anotações da medida da pressão arterial também na população idosa, questiona-se a manutenção da antiga crença de que o aumento da pressão arterial no idoso é físiológica e inócua. Está claro hoje, a eficácia do tratamento com redução dos riscos com controle da pressão arterial e a melhora da qualidade de vida dos hipertensos ${ }^{12}$

Em relação ao sexo, verificou-se que a medida da pressão arterial foi anotada nos pacientes do sexo masculino, em apenas um quarto, contra $45 \%$ nas mulheres. Embora se conheçam algumas diferenças nas variações da pressão arterial em ambos os sexos, esperava-se que as medidas fossem realizadas de maneira indistinta, causando surpresa a constatação dos valores apresentados.

Constatou-se ainda, que a pressão arterial não foi anotada, mesmo tendo referência de história familiar de hipertensão. A influência dos fatores genéticos na prevalência da hipertensão arterial é um fato que deve ser considerado. Sabe-se que a probabilidade de ser hipertenso quando se tem pai ou mãe hipertensos é de aproximadamente $50 \%$.

\section{CONCLUSÃo}

Os dados do presente estudo delimitam um quadro importante; a maioria dos prontuários (67\%) não tinha a pressão arterial anotada, ou seja, mais da metade das consultas analisadas, apesar de ter sido incluído na amostra prontuários de pacientes atendidos na Liga de Hipertensão onde necessariamente a pressão é medida e anotada; em $62 \%$ das consultas não constavam dados sobre história de hipertensão e dos pacientes que referiram história de hipertensão $(20 \%)$, nem todos tiveram a pressão arterial anotada; a pressão arterial foi anotada mais em pacientes do sexo feminino $(45 \%)$ do que no sexo masculino $(25 \%)$; e a pressão arterial foi anotada em $46 \%$ dos pacientes de 41 a 60 e em 33\% dos pacientes com mais de 60 anos. 
Portanto, os dados do presente estudo sugerem que a medida da pressão arterial não vem sendo realizada como preconiza a determinação do III Consenso Brasileiro de Hipertensão. Sugerem ainda, um elevado número de possiveis pacientes hipertensos privados dos cuidados necessários que podem estar contribuindo para a manutenção dos altos índices de morbi-mortalidade causados pela doença.

Considerando a alta prevalência da hipertensão e que a medida da pressão arterial é uma das maneiras mais rápida, segura, eficaz e se realizada corretamente, propicia dados indiscutiveis para a detecção precoce da doença, recomenda-se que:

- seja seguida a determinação do III Consenso Brasileiro de Hipertensão Arterial (1998) que preconiza a medida da pressão arterial em todas as consultas médicas independente da especialidade; $\mathrm{e}$

- que sejam realizadas campanhas enfatizando a necessidade da medida da pressão arterial. É importante que o enfermeiro esteja engajado nestas campanhas, considerando seu papel no âmbito da prevenção e detecção precoce da hipertensão arterial, mas também no que se relaciona ao ensino da população quanto à importância da medida periódica da pressão arterial.

\section{REFERÊNCIAS BIBLIOGRÁFICAS}

1. CONSENSO BRASILEIRO PARA O TRATAMENTO DA HIPERTENSÃO ARTERIAL, 3, São Paulo, C.B.H.A.B.G. Cultural, 1998.

2.PIERIN, A.M.G.; GOMES, M.A.M.; VEIGA, E.V.; NOGUEIRA, M.S.; NOBRE, F. Medida da pressão arterial no consultório e auto-medida da pressão: técnicas e equipamentos. In: MION Jr., D.; NOBRE, F. Medida da pressão arterial: da teoria à prática. 1.ed. São Paulo, Lemos, 1997. Cap.3, p. 35-65.

3. PARATI,G; POMIDOSSI, G. La mesurazione della pressione arteriosa: daí primi tentative al monitoraggio dinamico. Melo, Edizione Carlo Erba, 1988
4. ACHUTTI, A.C.; ACHUTTI,V.A.R. "Aspectos epidemiológicos" In: AMODEO, C; LIMA, E.G.; VASQUEZ, E.C. Hipertensão arterial. São Paulo, Sarvier, 1997. cap.2, p 11-22

5. LAURENTI,R. Epidemiologia da hipertensão arterial. In: CHIAVERINI,R. et al Hipertensão arterial para o clínico: etiopatologia, diagnóstico e tratamento. São Paulo, Atheneu, 1985.

6. BRASIL. Ministério da Saúde. Secretaria Nacional de Programas Especiais de Saúde. Divisão de doenças CrônicoDegenerativas. Programa Nacional de Educação e Controle da Hipertensão Arterial. Normas técnicas para o programa nacional de educação e controle da hipertensão arterial (PNECHA). Brasília. Centro de Documentação do Ministério da Saúde, 1988. (Série A: Normas e Manuais Técnicos,47).

7. COSTA,E.A. Hipertensão como problema de massa no Brasil: caracteres epidemiológicos e fatores de risco. Ci. Cult. v.35, n.11, p. 1642-1649, 1983.

8. LUNA, R.L. Hipertensão arterial. Rio de Janeiro, Medsi, 1989.

9. LESSA, I.; C CORTES, E.; MENEZES, G. A medida da pressão arterial na prática médica de rotina. Rev. Assoc. Med. Brasil, n. 30, p.52-54, 1984.

10. LESSA, M.C.N.; COSTA, M.E. Diferenças na medida da pressão arterial em primeiras consultas pré e pós implantação do Programa Nacional de Controle da Hipertensão. Rev. Assoc. Med. Brasil., v.39, n.3 p.141-5, 1993.

11. BRASIL. Ministério da Saúde. Secretaria Nacional de Assistência à Saúde. Coordenação de Doenças Cardiovasculares Doenças cardiovasculares no Brasil - Sistema Único de Saúde - SUS: dados epidemiológicos, assistência médica. Brasilia. 1993.

12. WAJNGARTEN, M.; SERRO-AZUL, J.B. Hipertensão em Idosos. In: AMODEO, C; LIMA, E.G.; VASQUEZ, E. C. Hipertensão arterial. São Paulo, Sarvier, 1997.cap. 26 p. 221-4. 


\section{ANEXO}

\section{Identificação}

Nome:

RG

Sexo F( ) M ( ) Idade anos

Cor Br. ( ) P( ) Mu () Ama()

Procedência:

Especialidade:

Medida da P.A

Valor I Valor II Valor III Valor IV

Posição sentado( ) em pé() deitado( ) não consta( )

Aparelho aneróide( ) coluna $\mathbf{H g}($ )

Antecedentes Hipertensão Arterial

Sim( ) Não( ) Não consta( ) 